University of Wollongong

Research Online

Faculty of Engineering - Papers (Archive)

Faculty of Engineering and Information

Sciences

2006

\title{
Absolute depth-dose-rate measurements for an 192Ir HDR brachytherapy source in water using MOSFET detectors
}

\author{
V. O. Zilio \\ Ecole Polytechnique Federale de Lausanne, Switzerland \\ O. P. Joneja \\ Ecole Polytechnique Federale de Lausanne, Switzerland \\ Y. Popowski \\ University of Geneva Hospital, Switzerland \\ Anatoly B. Rosenfeld \\ University of Wollongong, anatoly@uow.edu.au \\ R. Chawla \\ Ecole Polytechnique Federale de Lausanne, Switzerland
}

Follow this and additional works at: https://ro.uow.edu.au/engpapers

Part of the Engineering Commons

https://ro.uow.edu.au/engpapers/145

\section{Recommended Citation}

Zilio, V. O.; Joneja, O. P.; Popowski, Y.; Rosenfeld, Anatoly B.; and Chawla, R.: Absolute depth-dose-rate measurements for an 192Ir HDR brachytherapy source in water using MOSFET detectors 2006.

https://ro.uow.edu.au/engpapers/145

Research Online is the open access institutional repository for the University of Wollongong. For further information contact the UOW Library: research-pubs@uow.edu.au 


\title{
Absolute depth-dose-rate measurements for an ${ }^{192}$ Ir HDR brachytherapy source in water using MOSFET detectors
}

\author{
Valéry Olivier Zilio \\ Ecole Polytechnique Fédérale de Lausanne (EPFL), Laboratoire de Physique des Réacteurs et de \\ Comportement des Systèmes, CH-1015, Lausanne, Switzerland \\ Om Parkash Joneja ${ }^{\text {a) }}$ \\ Ecole Polytechnique Fédérale de Lausanne (EPFL), Laboratoire de Physique des Réacteurs et de \\ Comportement des Systèmes, CH-1015, Lausanne, Switzerland and Paul Scherrer Institute (PSI), \\ Laboratory of Reactor Physics and Systems Behaviour, CH-5232 Villigen, Switzerland \\ Youri Popowski \\ Radio-oncology Division, University of Geneva Hospital, CH-1211, Genève, Switzerland \\ Anatoly Rosenfeld \\ Centre for Medical Radiation Physics, University of Wollongong, Wollongong, NSW, 2522 Australia \\ Rakesh Chawla \\ Ecole Polytechnique Fédérale de Lausanne (EPFL), Laboratoire de Physique des Réacteurs et de \\ Comportement des Systèmes, CH-1015, Lausanne, Switzerland and Paul Scherrer Institute (PSI), \\ Laboratory of Reactor Physics and Systems Behaviour, CH-5232 Villigen, Switzerland
}

(Received 5 April 2005; revised 21 March 2006; accepted for publication 24 March 2006; published 9 May 2006)

Reported MOSFET measurements concern mostly external radiotherapy and in vivo dosimetry. In this paper, we apply the technique for absolute dosimetry in the context of HDR brachytherapy using an ${ }^{192}$ Ir source. Measured radial dose rate distributions in water for different planes perpendicular to the source axis are presented and special attention is paid to the calibration of the $\mathrm{R}$ and $\mathrm{K}$ type detectors, and to the determination of appropriate correction factors for the sensitivity variation with the increase of the threshold voltage and the energy dependence. The experimental results are compared with Monte Carlo simulated dose rate distributions. The experimental results show a good agreement with the Monte Carlo simulations: the discrepancy between experimental and Monte Carlo results being within 5\% for $82 \%$ of the points and within $10 \%$ for $95 \%$ of the points. Moreover, all points except two are found to lie within the experimental uncertainties, confirming thereby the quality of the results obtained. (C) 2006 American Association of Physicists in Medicine. [DOI: 10.1118/1.2198168]

Key words: MOSFET, absolute dosimetry, Monte Carlo, brachytherapy, ${ }^{192}$ Ir source

\section{INTRODUCTION}

Patient or in vivo dosimetry requires small size radiation detectors having response proportional to the dose delivered during the course of irradiation. There are currently four main methods of radiotherapy dosimetry viz. thermoluminescent dosimeter (TLD), diode, metal-oxide semiconductor field-effect transistor (MOSFET), and film. The TLD and film methods are passive in nature with the former requiring an elaborate heating arrangement and a photomultiplier set up for measuring doses. The major disadvantages for the TLD and film methods are time-consuming calibration, offline measurement, and external data treatment to determine dose rates. Diodes on the other hand are a semiconductor analogy of ionization chambers, somewhat larger in size than TLDs. A radiotherapy diode in principle operates the same way as a photodiode, with current proportional to dose rate and total integrated charge to absorbed dose. Diodes are simpler to use than TLDs and are able to provide on-line dosimetry. Their major disadvantages are the size and the several correction factors required for application in clinical radio- therapy. MOSFET offers the advantages of a smaller size over the other dosimeters as well as fewer correction factors compared to usual diodes. Radiation absorbed in the sensitive volume underneath the gate of a MOSFET results in a permanent change in its threshold voltage, which is found to be proportional to the absorbed dose. The response of the MOSFET dosimeter is measured by threshold voltage change, which is quite sensitive to the gate bias during irradiation.

MOSFETs are reported to have been used in the context of standard external beam radiation therapy, ${ }_{3}^{1}$ intensity modulated radiation therapy, ${ }^{2}$ in vivo dosimetry, ${ }^{3-5}$ image guided radiotherapy, ${ }^{6}$ microbeam radiotherapy, ${ }^{7}$ and diagnostic radiology. ${ }^{8}$ Such measurements are either carried out at energies well above $300 \mathrm{keV}$ (where the response remains fairly constant) or under nonvarying photon spectrum conditions.

MOSFET dosimeters offer several advantages over conventional radiation detectors, which include their submillimeter size, immediate re-use capability, and nondestructive readout. On the other hand, they have certain important dis- 


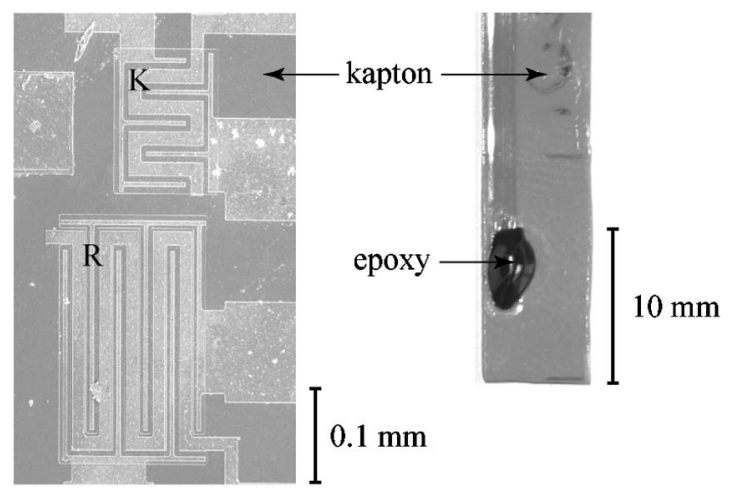

FIG. 1. The R and K sensors of a single MOSFET chip (left side) along with an epoxy bead covering both the sensors (right side).

advantages over the other detectors mentioned earlier, viz. a strong energy dependence of their response below $300 \mathrm{keV}$ in a photon field, similar to a filter free Si diode, and sensitivity variation with the increase of the threshold firing voltage $\left(V_{\text {th }}\right)$.

In this paper, we have addressed the above-indicated shortcomings of MOSFET detectors and have applied them for absolute dosimetry in the context of HDR brachytherapy using an ${ }^{192} \mathrm{Ir}$ source, for comparable absorbed dose rates as in the actual treatment. Measured radial dose rate distributions in water for different planes perpendicular to the source axis are presented. Special attention has been paid to the calibration of the detectors and the determination of appropriate correction factors via measurements employing tiny, commercially available ionization chamber and Monte Carlo simulations using the MCNP4C code. ${ }^{9}$ Finally, a detailed uncertainty analysis of the experimental data, based on the "Guide to the Expression of Uncertainty in Measurements (GUM)" published by ISO,${ }^{10}$ is presented to confirm the quality of the results obtained.

\section{MATERIALS AND METHODS}

\section{A. Detectors and experimental setup}

As shown in Fig. 1, each MOSFET chip contains two sensors, referred to as $\mathrm{R}$ and $\mathrm{K}$ type, respectively, placed one over the other on a Kapton substrate and protected under epoxy resin. The two sensors are independent and the high sensitivity $\mathrm{R}$ type has a thicker gate oxide than the $\mathrm{K}$ sensor. More details about the sensors and their topology can be found in Ref. 11. The sensitive area of the $\mathrm{R}$ and $\mathrm{K}$ type sensors are $160 \times 260$ and $120 \times 150 \mu \mathrm{m}^{2}$, respectively. The horizontal distance between the centers of the two sensors is about $20 \mu \mathrm{m}$, and the physical separation in the vertical direction is about $40 \mu \mathrm{m}$, with the $\mathrm{R}$ sensor located at the bottom of the Kapton carrier. Thus, from the viewpoint of the present measurements, the $\mathrm{R}$ and $\mathrm{K}$ sensors can be considered as lying at the same location, and there is no need for separate alignment of each sensor with respect to the source center. The chip is wire bonded on the Kapton pigtail with gold plated connectors. The other end is welded to a suitable six-pin connector and finally connected by a special cable to the reader.

Commercially available MOSFET probe, RADFET chip type TOT 500 designed and fabricated by REM Oxford, UK and clinical MOSFET semiconductor dosimetry system used, ${ }^{11}$ were developed and supplied by the Centre for Medical Radiation Physics (CMRP), University of Wollongong, NSW, Australia, under the bilateral CMRP/EPFL collaboration on dosimetric applications. The dosimetry system allows five probes to be connected and is capable of reading both the sensors in a given probe at the same time with the help of a toggle switch or via a dedicated PC. The MOSFETS were used in real-time mode with a positive bias applied to the gate to improve their linearity. The gate bias was much lower for the $\mathrm{R}$ than the $\mathrm{K}$ sensor and their response was linear up to 50 and $200 \mathrm{~Gy}$, respectively. Standard readout equipment and method ${ }^{11}$ for such detectors was used which involved measurement of change in the threshold voltage under constant current between source and drain. The energy response of such detectors depends upon the aluminum gate, epoxy material apart from the photon interaction cross section with the $\mathrm{SiO}_{2}$. The sensitivity of the $\mathrm{R}$ and $\mathrm{K}$ sensors were about 5 and $0.15 \mathrm{mV} / \mathrm{Gy}$, respectively. The uncertainty associated with the reading equipment was $1 \mathrm{mV}$ and in the real-time mode, the instability has been found to be lower then $\pm 2 \mathrm{mV}$ (coverage factor, $k=1$ ) before and after the irradiation. In fact, special efforts were made to reduce the effect of radiation induced instabilities. Angular response of similar detectors have been studied previously ${ }^{12}$ and found to be isotropic within about $2 \%-3 \%$.

Measurements of dose rate distributions with MOSFET have been carried out in a water phantom, which has the advantage of being near tissue equivalent, homogeneous, cheap, and easy to characterize in terms of composition and density. In addition, the distance between source and dosimeter can be varied continuously. The phantom consisted of a $40 \mathrm{~cm}$ cubic plastic tank filled with water. A metallic structure was set up on top of the tank to support two 8-cm range, high-precision translation stages. Special holders were developed to clamp the source and the detector to the translation stage, so that the distance between the two could be measured accurately. The old microSelectron ${ }^{192}$ Ir HDR Classic source [Nucletron B. V., Veenendaal, The Netherlands] was held in a brachytherapy $\mathrm{Ti}-\mathrm{Zr}$ needle having an inner and outer diameter of 1.4 and $1.9 \mathrm{~mm}$, respectively. For delivering the HDR source to the preselected locations, a remotely controlled Nucletron afterloader machine was used. All the detector positions were selected sufficiently far from the tank walls and external surrounding $(15-20 \mathrm{~cm})$ to avoid any interference. The MOSFETs were kept within a thin waterproof plastic holder. The experimental arrangement without the reader is shown in Fig. 2.

\section{B. Monte Carlo simulations}

The Monte Carlo code MCNP4C along with its associated data library DLC $200^{9}$ has been used for the numerical simu- 


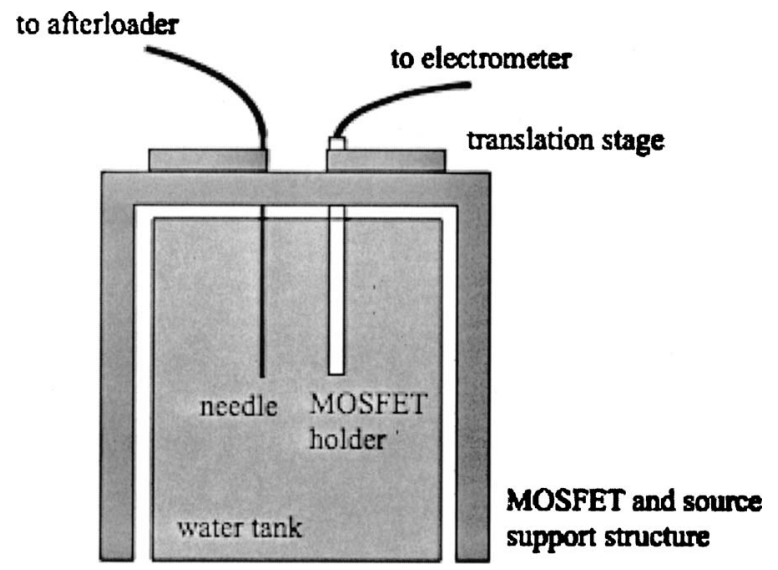

FIG. 2. Experimental setup.

lations, calculations being performed currently on a PC working in a Microsoft Windows environment. A detailed model of the ${ }^{192}$ Ir HDR source used has been developed using data derived from Refs. 13 and 14. The detailed physics option of the code has been selected for the calculations, which include coherent (Thomson) scattering and accounts for the fluorescent photons after photoelectric absorption. Atomic form factors and incoherent functions are used with coherent and incoherent scattering, respectively, to account for electron binding effects. Neither of the two detectors was simulated, and it was assumed that the electron equilibrium was maintained at all locations considered and hence the dosimetric behavior could be well predicted by considering photon transport only i.e., the recoil kinetic energy of the electron is deposited locally. Furthermore, due to the azimuthal symmetry around the source, the F5 ring tally, which is an extension of the point detector tally and provides far lower statistical error for a given number of histories, was used in conjunction with the tally multiplier card $F m$, to directly calculate, dose values $\left(\mathrm{cGy} \mathrm{Bq}^{-1} \mathrm{~h}^{-1}\right)$ in water at all the experimental locations, in the planes transverse to the source axis. The default photon cutoff limit of $1 \mathrm{keV}$ was selected for all calculations. For calculating average photon energy at different experimental locations photon energy fluence tally with a small energy bin of $1 \mathrm{keV}$ was employed. For each run, 10 million histories were simulated in order to have a statistical uncertainty (coverage factor, $k=1$ ) lower than $1 \%$ at all points.

\section{MOSFET calibration and correction factors}

A MOSFET detector acts as a radiation dosimeter because the change in its threshold voltage $\left(V_{\text {th }}\right)$ to first approximation is proportional to the dose delivered in the oxide layer. ${ }^{15}$ The rate of shift in threshold voltage $\Delta \dot{V}$ for a $1 \mathrm{MBq}$ source is measured in $\mathrm{mV} \mathrm{h}^{-1}$, and the corresponding dose rate is then determined from the knowledge of a calibration factor. The latter factor $\left(f_{C}\right)$ is expressed in $\mathrm{cGy} \mathrm{mV}^{-1}$ and is measured under reference conditions (see Sec. II C 1). However, there are some other factors that need to be considered before the absolute dose rate, under given experimental conditions, can be determined from the measured response. For the type of MOSFETs used, it has been reported ${ }^{16}$ that the change in sensitivity due to variation in the ambient temperature is insignificant if the initial and final readout conditions remain the same, this being the case for the present measurements. Thus, no temperature correction factor has been applied. There are, however, two other important correction factors that need to be considered. The first, $f_{S}(V)$, accounts for the sensitivity variation with the increase of the $V_{\text {th }}$, and the second, $f_{E}(d)$, accounts for the energy dependence of the MOSFET response, which in turn depends upon the distance of penetration " $d$ " of the radiation in water.

In brief, the absolute dose rate in $\mathrm{cGy} \mathrm{h}^{-1}$ for a $1 \mathrm{MBq}$ activity source can be obtained by applying the following relation:

$$
\begin{aligned}
\dot{D}\left[\mathrm{cGy} \mathrm{MBq}^{-1} \mathrm{~h}^{-1}\right]= & \Delta \dot{V}\left[\mathrm{mV} \mathrm{MBq}^{-1} \mathrm{~h}^{-1}\right] f_{C}\left[\mathrm{cGy} \mathrm{mV}^{-1}\right] \\
& \times f_{S}(V) f_{E}(d) .
\end{aligned}
$$

\section{Calibration procedure}

It is reasonable to assume that the rate of shift in threshold voltage $\Delta \dot{V}$ is proportional to the dose rate $\dot{D}$ if the change in $V_{\text {th }}$ of the MOSFET is within a few hundred millivolts. For determining an appropriate calibration factor for each sensor, a reference point at a distance of $10 \mathrm{~mm}$ in the central plane was selected. The dose rate in water at the reference point was then measured by using an accurately calibrated $0.22 \mathrm{~cm}^{3}$ ionization chamber, model 2577C from NE SaintGobain Crystals and Detectors UK Ltd. The standard calibration of the chamber in terms of air KERMA in air protocol was carried out using ${ }^{137} \mathrm{Cs}$ sources at Paul Scherrer Institute (PSI) and at the Institute de Radiophysique Appliquée (IRA), and with a ${ }^{60} \mathrm{Co}$ source at IRA. The values obtained at IRA are traceable to NPL primary standard and additional confirmation is obtained via PSI measurements traceable to PTWFreiburg, Germany. The values obtained were transformed to dose absorbed in water by application of suitable correction factors. ${ }^{17,18}$ These include corrections due to range of electrometer, ambient temperature and pressure conditions, energy, material, polarity, and charge recombination. It should be mentioned that detailed validation of MCNP dosimetric values, for a routinely used PLATO-BPS system for brachytherapy procedure and for dosimetry with a ${ }^{144} \mathrm{Ce}$ source, have been reported in Refs. 14 and 18, respectively. The dose rate value $\dot{D}_{\text {ref }}$ thus obtained for contained activity was found to be $0.106 \pm 0.001 \mathrm{cGy} \mathrm{MBq}{ }^{-1} \mathrm{~h}^{-1}$ at the reference point. The calibration factor for each sensor type could then be determined by substituting the response of the respective sensor at the reference location $\left(\Delta \dot{V}_{\text {ref }}\right)$ in the following:

$$
f_{C}=\frac{\dot{D}_{\text {ref }}}{\Delta \dot{V}_{\text {ref }} \cdot f_{S}\left(\dot{V}_{\text {ref }}\right) \cdot f_{E}\left(d_{\text {ref }}\right)},
$$

where $f_{S}\left(V_{\text {ref }}\right)$ and $f_{E}\left(d_{\text {ref }}\right)$ represent the sensitivity and energy correction factors at the reference point as described in the following. 


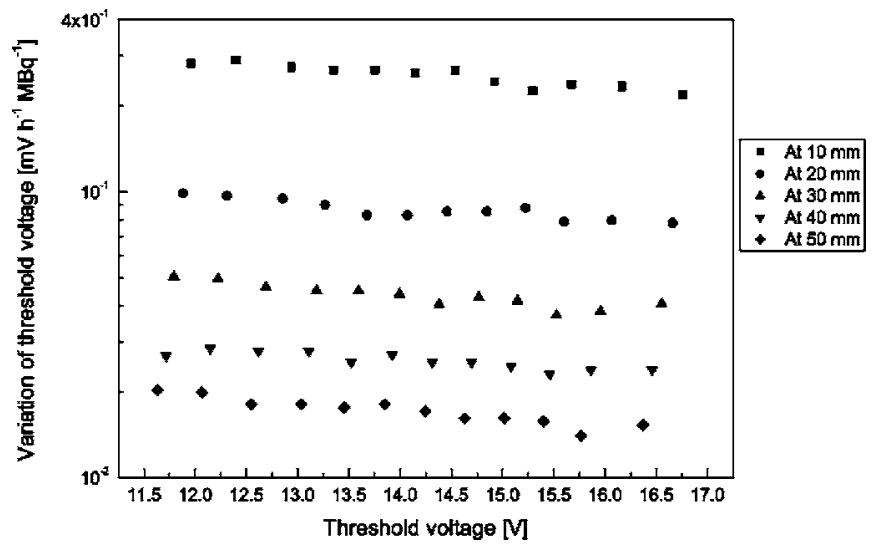

FIG. 3. Sensitivity studies for an R type detector as a function of $V_{\mathrm{th}}$.

In order to determine the calibration factor, exposure times after correcting for source transit were adjusted to give a shift in the $V_{\text {th }}$ of about $50 \mathrm{mV}$, for which the reading uncertainty (coverage factor, $k=1$ ) is about $2 \mathrm{mV}$ or $4 \%$. Calibration runs were carried out at the beginning and at the end of all measurements and the mean $\Delta \dot{V}$ values, corrected with $f_{S}\left(V_{\text {ref }}\right)$ and $f_{E}\left(d_{\text {ref }}\right)$, were used to determine the calibration factor from Eq. (2). It may be noted that this method automatically includes the correction due to the structural and packaging materials of the MOSFET.

\section{Sensitivity variation with the increase of the $V_{\text {th }}$}

The sensitivity variation with the increase of the $V_{\text {th }}$ due to the accumulated dose has been studied for both $\mathrm{R}$ and $\mathrm{K}$ type of MOSFETs at distances ranging from 5 to $50 \mathrm{~mm}$ from the HDR source in the plane perpendicular to the source axis and passing through its center, i.e., the central plane. Twelve and six successive distributions were recorded for $\mathrm{R}$ and $\mathrm{K}$ type detectors, respectively, while maintaining a threshold shift between 30 and $60 \mathrm{mV}$. A subset of measured values for an $\mathrm{R}$ type sensor showing changes in $V_{\text {th }}$ $\left(\mathrm{mV} \mathrm{h}^{-1} \mathrm{MBq}^{-1}\right)$ versus the $V_{\text {th }}(V)$ are depicted in Fig. 3. The latter parameter $\left(V_{\mathrm{th}}\right)$ could also be considered as a measure of accumulated dose if the calibration factor $f_{C}$ were not depending on it.

The measured distributions have been fitted with linear functions and the mean slope employed to estimate the sensitivity variation. The sensitivity reduction $L$ was found to be $0.045 \pm 0.008$ and $0.035 \pm 0.003$ per volt increase in the threshold voltage for $\mathrm{R}$ and $\mathrm{K}$ type detectors, respectively. The sensitivity correction factor $f_{S}(V)$ for a given threshold voltage $V$ in volts is then calculated from

$$
f_{S}(V)=1+L \cdot\left(V-V_{I}\right),
$$

where $V_{I}$ denotes the initial threshold voltage of the MOSFET.

\section{Energy dependence}

MOSFET detectors have photon energy response ${ }^{19-21}$ similar to the other silicon sensors which are driven by the photoelectric effect cross sections of silicon and the charge recombination in the gate oxide and packaging of the chip. This response, however, is well known to be nonlinear, especially for energies below $300 \mathrm{keV}$. Thus, the normalized MOSFET response to a $6 \mathrm{MV}$ white $\mathrm{x}$-ray spectrum from a medical linear accelerator shows a significant increase with the decrease of photon energy. It attains a maximum value at about $40 \mathrm{keV}$ and falls down rapidly on either side, attaining a fairly constant value at energies above about $300 \mathrm{keV}$. The observed energy response for energies between 1 and $20 \mathrm{keV}$ has been attributed to the recombination of electron-hole pairs in the silicon oxide. ${ }^{17}$ The variation at energies from 20 to $100 \mathrm{keV}$ is explained on the basis of a dominant contribution from the photoelectric effect in $\mathrm{SiO}_{2}$ (in comparison with tissue). In HDR brachytherapy using ${ }^{192} \mathrm{Ir}$, the average photon energy at larger depths of penetration decreases, resulting in a change in the secondary electron spectra, and therefore a nonlinear response of the MOSFET in the photon field. It is thus important to determine the appropriate value for the energy dependence of the MOSFET response, i.e., the factor $f_{E}\left(d_{\text {ref }}\right)$, with sufficient accuracy.

As reported by Kron et al., ${ }^{20}$ the detector response is found to vary considerably with the incident photon energy. After reaching a maximum value between 40 and $50 \mathrm{keV}$, the response of MOSFET is found to reduce exponentially for lower energies. At medium energies, as already indicated, the dependence is mainly governed by the photoelectric effect and at higher energies it remains fairly constant. For materials of low atomic number, the cross section for the photoelectric effect is approximately inversely proportional to the cube of the photon energy and hence one can write:

$$
W(E)=\left[1-e^{\left(-a_{1}\left(E-E_{1}\right)\right)}\right]\left[1+\frac{a_{2}}{\left(E-E_{2}\right)^{3}}\right],
$$

where $a_{1}$ and $a_{2}$ are two fitting parameters which determine the importance of exponential and inverse cubic falls at low and medium energies, respectively. $E_{1}$ and $E_{2}$ allow for an energy shift of the two components. In practice, the variation of the energy response $W(E)$ means that with the increase in the distance between the source and detector, the MOSFET reading $\left(\mathrm{mV} \mathrm{h}^{-1}\right)$ is larger, because of the softening of the photon energy spectrum of ${ }^{192} \mathrm{Ir}$ in water. The energy correction factor $f_{E}(d)$ is thus defined as the inverse of the energy response $W(E)$. This model is normalized to a high photon energy from a linear accelerator where the response is flat. In this work, the correction factor is essentially determined from the ratio of the response at a distance " $d$ " relative to that at the reference point.

In the current studies, the energy response of the $\mathrm{R}$ and $\mathrm{K}$ type detectors was not measured experimentally. Instead, a fit was made of the experimental response values of Kron et $a^{20}{ }^{20}$ (obtained with mono-energetic synchrotron radiation and X-ray spectra between 10 and $6000 \mathrm{keV}$ for an $\mathrm{R}$ type MOSFET, similar in respect of oxide thickness, gate, and epoxy material to the one employed in our studies) and the same was considered valid for $\mathrm{K}$ sensor. This is a fairly good approximation because both $\mathrm{R}$ and $\mathrm{K}$ sensors are produced 


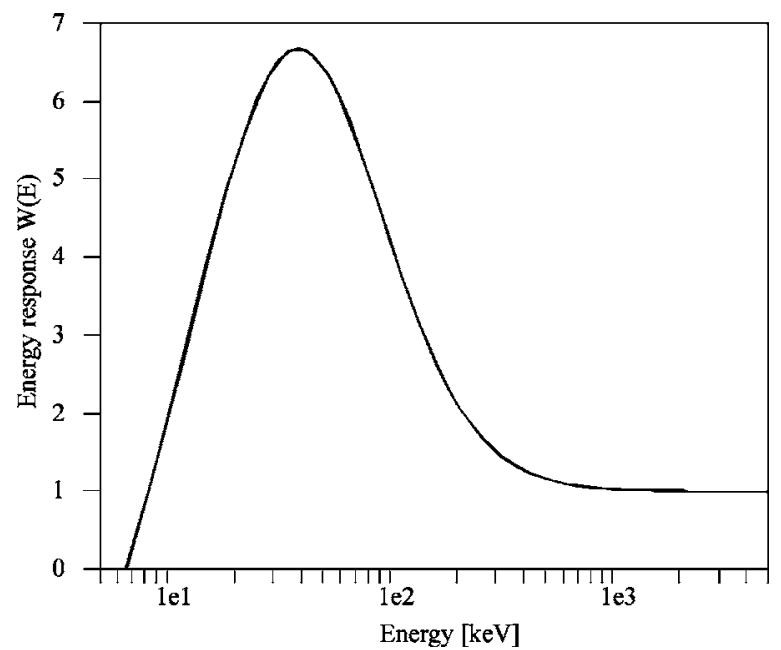

FIG. 4. Energy response of R type MOSFET detector normalized to unity for $6 \mathrm{MV}$ spectral $\mathrm{x}$ rays from a medical linear accelerator (from Kron $e t$ al.-Ref. 20).

on the same chip and via the same technological process. Furthermore, difference in silicon oxide layer thickness between $\mathrm{R}$ and $\mathrm{K}$ sensors and thereby associated electrical field effects, influence the recombination rate of electron hole pairs for energies far lower than that of the current interest. ${ }^{22,23}$ Knowing that, relative to a response of unity at higher energies (above $400 \mathrm{keV}$ ), the response at $40 \mathrm{keV}$ is 6.6 (as per Kron $e t a l^{20}$ ), the fitting parameters obtained for Eq. (4) are $a_{1}=0.04, a_{2}=4.03 \times 10^{7}, E_{1}=7$, and $E_{2}=-130$. The energy response obtained with these fitting parameters is shown in Fig. 4.

In order to determine appropriate energy dependent correction factors for gamma sources from Fig. 4, the mean energy of the photon spectrum at each experimental location has been considered to represent dose, an approximation found reasonable in the case of ${ }^{192} \mathrm{Ir}$ source because the dominant dose contributions at distances considered arise from photons beyond $100 \mathrm{keV}$. In this context, MCNP calculations have been performed to determine the evolution of the photonic energy spectrum at distances ranging from the surface of the ${ }^{192} \mathrm{Ir}$ source to a distance of $50 \mathrm{~mm}$ in water. Concentric cylinders of the same height as the active section of the ${ }^{192} \mathrm{Ir}$ source $(3.5 \mathrm{~mm})$ and an energy bin of $1 \mathrm{keV}$ were considered for the calculating photon energy fluence by F5 ring tally. The mean energies found at the experimental locations (see Table I), were used to determine the energy response correction factor $f_{E}(d)$ at various distances between detector and source relative to the flat response region at higher photon energy. For correction factors at locations other than in the central plane, the mean photon energy values were interpolated from the results for calculated mean energy versus distance.

\section{Experimental procedure}

First, the multilead cable and connectors were suitably shielded from the electromagnetic noise. The MOSEFT detector was then placed at the reference position and con-
TABLE I. Energy correction factor at different distances in a water phantom.

\begin{tabular}{ccc}
\hline \hline Distance $[\mathrm{mm}]$ & Mean energy $(\mathrm{keV})$ & $f_{E}(d)=W^{-1}(E)$ \\
\hline 5 & 340.32 & 0.72 \\
10 & 323.55 & 0.70 \\
15 & 308.08 & 0.68 \\
20 & 293.91 & 0.65 \\
25 & 281.04 & 0.63 \\
30 & 269.47 & 0.61 \\
35 & 259.20 & 0.59 \\
40 & 250.23 & 0.58 \\
45 & 242.56 & 0.56 \\
50 & 236.19 & 0.55 \\
\hline \hline
\end{tabular}

nected to the reader. It was left undisturbed for about $30 \mathrm{~min}$ to achieve stable working conditions. The initial threshold voltage was recorded at each point for the sensitivity correction factor, and a calibration measurement was performed five times at the standard reference position. Similarly, after measurements at all distances, five additional runs of calibration at the reference position were carried out. The mean calibration factor was determined, as described earlier.

For dose distribution measurements in different planes, the MOSFET was held fixed and the source moved in the axial and transverse directions to find the source centre. Later, measurements were carried out at different distances in the source bisecting (central plane) and other planes by suitably moving the MOSFET and always transporting the source to the same reference location. Measurements were carried out radially, from 5 to $50 \mathrm{~mm}$, in steps of $5 \mathrm{~mm}$, in each plane situated at a height ranging from 0 to 25 from the central plane (for example, plane 10 in Fig. 5 is located at a height of $10 \mathrm{~mm}$ from the central plane). In order to have measurement at different radial positions, the MOSFET was moved step-wise starting from the furthest position from the source, and once the detector reached the correct position, the source was moved into the desired position for a preselected time of irradiation.

\section{RESULTS AND DISCUSSION}

\section{A. Results of the radial dose rate measurements}

The measured dose rate distributions, as obtained with both $\mathrm{R}$ and $\mathrm{K}$ type MOSFET sensors (two of R type, i.e., R2 and $\mathrm{R} 3$ and one of $\mathrm{K}$ type, i.e., $\mathrm{K} 2$ ) in the different planes are presented in Fig. 5, along with the Monte Carlo simulated results. Also indicated, in each case, is the discrepancy $E$ between the mean experimental value $\dot{D}_{\mathrm{EX}}$ and the corresponding Monte Carlo result $\dot{D}_{\mathrm{MC}}$ (calculated using ring tally 5), i.e., as determined from

$$
E=100 \cdot\left(1-\frac{\dot{D}_{\mathrm{EX}}}{\dot{D}_{\mathrm{MC}}}\right) .
$$

The mean experimental results show a good agreement with the Monte Carlo simulations, the discrepancy between experimental and Monte Carlo results being within 5\% for 

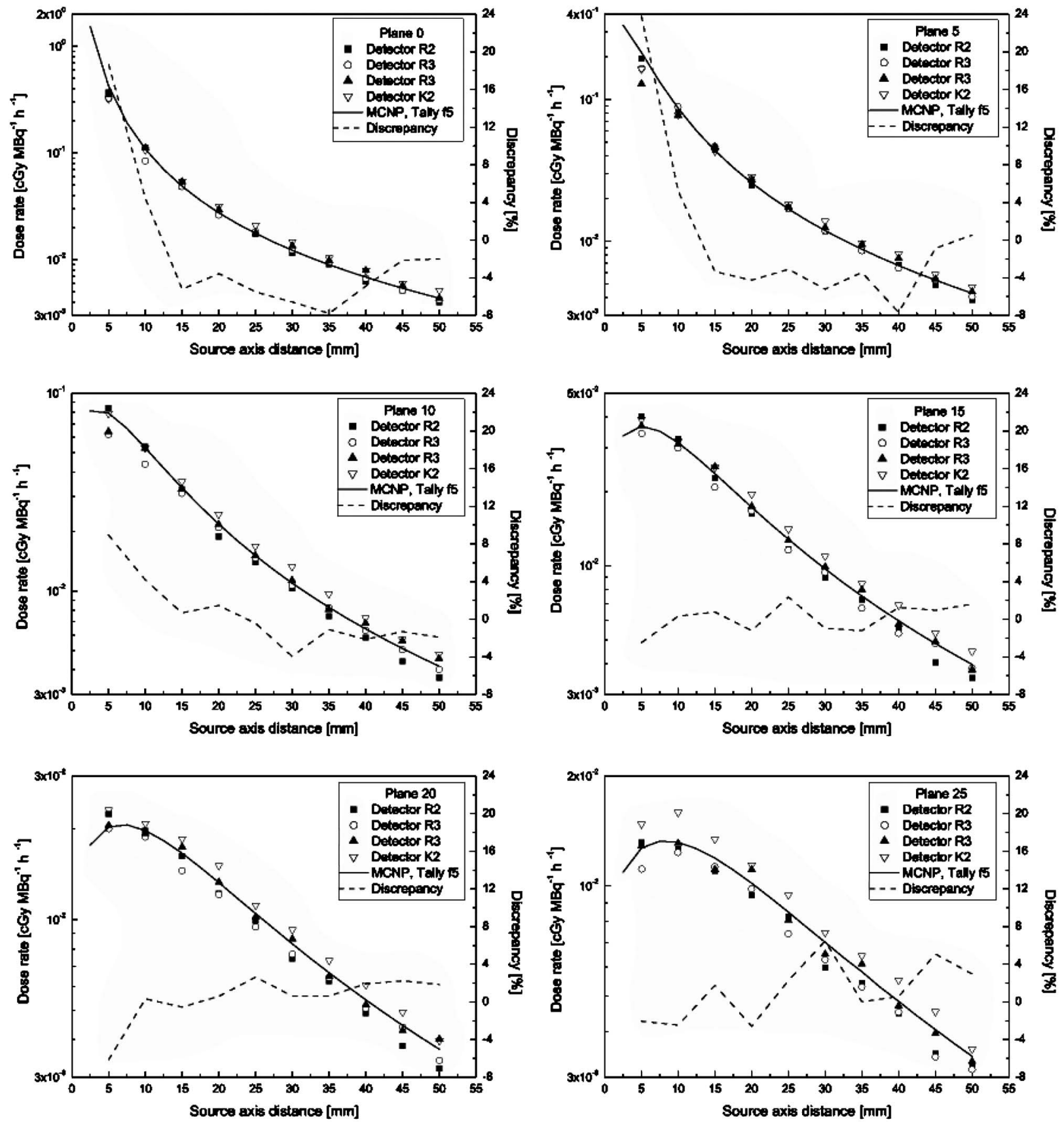

FIG. 5. Comparison of measured and calculated radial dose rate distributions around an ${ }^{192}$ Ir HDR source placed in a water phantom. The discrepancies indicated are with respect to mean experimental values.

$82 \%$ of the points and within $10 \%$ for $95 \%$ of the points. Moreover, all points except two are found to lie within the experimental uncertainties presented in the next section.

Generally, discrepancies close to the source are found to be positive. This could be due to a systematic effect on the response of the MOSFET due to the large variation in the dose rates with small changes of distance. Unlike in the case of LINAC irradiations, the dose rates in the current measurements vary from close to distant locations by about a factor of 100. The accuracy of the MOSFET dosimetry may have been affected by slow fading due to the much longer expo- sure time which was required for a statistically reliable $\Delta \dot{V}$ at larger distances. This effect can explain the difference in the sign of the discrepancy between the Monte Carlo simulations and the experiments in most situations. Low sensitivity is clearly a limitation for the application of real time MOSFET dosimetry for HDR in vivo brachytherapy, when the distance between sources and detectors become larger than $40 \mathrm{~mm}$.

Furthermore, it is seen that, at larger distances, the values measured with the $\mathrm{R}$ sensor are somewhat lower than calculated, while the $\mathrm{K}$ detector results are greater. This could 
partly be due to the use of the same energy dependent correction factor for the $\mathrm{R}$ and $\mathrm{K}$ sensors as was derived from experimental factor for $\mathrm{R}$ sensor. Electrical field in a thinner gate oxide in case of $\mathrm{K}$ sensor was about ten times larger, leading thereby to smaller recombination of charge with softening of the photon spectra in comparison with $\mathrm{R}$ sensor making relative response higher for the $\mathrm{K}$ type sensor.

\section{B. Uncertainty analysis for measured dose rates}

In order to estimate the uncertainty on the measured dose rates, one clearly needs to consider uncertainties for each of the four components on the right-hand side in Eq. (1). The current analysis, as mentioned earlier, is based on GUM representation and restricted to a confidence level of 68\% (coverage factor $k=1$ ). The uncertainties listed as type A are obtained by statistical analysis, whereas those of type B depend on considerations other than statistical. All components are assumed to be noncorrelated.

As per Eq. (2), the type A uncertainty of the calibration factor depends on the uncertainties on the reference dose rate, the shift in threshold voltage, and the ones of the sensitivity and energy correction factors. All contributions must be added in quadrature, following the law of propagation of uncertainties. The type A uncertainty on the reference dose rate determined from the standard calibrated ionization chamber has been assessed as being $1 \%$. The corresponding values due to the variation of threshold voltage as $4 \%$ and $2 \%$ for $\mathrm{R}$ and $\mathrm{K}$ type detectors, respectively. The uncertainty due to sensitivity variation as determined from the change in slopes obtained from the linear fits, as $5.6 \%$ and $8.7 \%$ for $\mathrm{R}$ and $\mathrm{K}$ type detectors, respectively. The type $\mathrm{B}$ uncertainty on the energy correction factor is estimated to be $5 \%$ due to the combined effect of errors arising from curve fitting and mean energy consideration compared to the full spectrum. Thus, the net uncertainty on the calibration factor works out to be $8.6 \%$ and $10.8 \%$ for $\mathrm{R}$ and $\mathrm{K}$ type MOSFETs, respectively.

From the calibration certificate of the ${ }^{192} \mathrm{Ir}$ source, the type B uncertainty on the air kerma rate $u(K)$, used to deduce
TABLE II. Relative uncertainties on the measured dosimetric values for an ${ }^{192}$ Ir HDR source at different distances from the source at center plane, using $\mathrm{R}$ and $\mathrm{K}$ sensors of MOSFET dosimeters.

\begin{tabular}{|c|c|c|c|c|c|c|}
\hline \multirow[b]{3}{*}{ Component } & \multirow[b]{3}{*}{ Type A (\%) } & \multicolumn{5}{|c|}{$\mathrm{R}$ type sensor } \\
\hline & & \multicolumn{5}{|c|}{$\begin{array}{c}\text { Type B (\%) } \\
\text { distance }(\mathrm{mm})\end{array}$} \\
\hline & & 10 & 20 & 30 & 40 & 50 \\
\hline$u(\Delta V)$ & 4 & & & & & \\
\hline$u\left(f_{c}\right)$ & 8.6 & & & & & \\
\hline$u\left(f_{s}\right)$ & 5.6 & & & & & \\
\hline$u\left(f_{E}(d)\right)$ & & 5 & 5 & 5 & 5 & 5 \\
\hline$u(K)$ & & 1.7 & 1.7 & 1.7 & 1.7 & 1.7 \\
\hline$u(P)$ & & 9 & 5 & 3 & 2 & 2 \\
\hline$u(D)_{A, B}$ & 11 & 10.4 & 7.3 & 6.1 & 5.6 & 5.6 \\
\hline$u(D)_{t o t}$ & & 15.2 & 13.2 & 12.6 & 12.4 & 12.4 \\
\hline \multicolumn{7}{|c|}{ K type sensor } \\
\hline$u(\Delta V)$ & 2 & & & & & \\
\hline$u\left(f_{c}\right)$ & 10.8 & & & & & \\
\hline$u\left(f_{s}\right)$ & 8.7 & & & & & \\
\hline$u\left(f_{E}(d)\right)$ & & 5 & 5 & 5 & 5 & 5 \\
\hline$u(K)$ & & 1.7 & 1.7 & 1.7 & 1.7 & 1.7 \\
\hline$u(P)$ & & 9 & 5 & 3 & 2 & 2 \\
\hline$u(D)_{A, B}$ & 14 & 10.4 & 7.3 & 6.1 & 5.6 & 5.6 \\
\hline$u(D)_{t o t}$ & & 17.5 & 15.8 & 15.3 & 15.1 & 15.1 \\
\hline
\end{tabular}

the contained activity of the source, is $1.7 \%$. Furthermore, the error on the distance between the source center and the detector is 0.4 and $0.3 \mathrm{~mm}$ in the radial and axial directions, respectively. The error estimates take into account the movement of the source and MOSFET in their respective holding arrangements, uncertainties associated with translation stages, and shape of the source needle. Using the inverse square law, one finds that the corresponding uncertainties due to positioning $u(P)$ range from $9 \%$ at $10 \mathrm{~mm}$ to $2 \%$ at $50 \mathrm{~mm}$. The global relative uncertainty on the dose rate is calculated from Eq. (6), and the corresponding values obtained for both types of detectors are summarized in Table II,

$$
\frac{u(\dot{D})}{\dot{D}}=\sqrt{\left(\frac{u(\Delta \dot{V})}{\Delta \dot{V}}\right)^{2}+\left(\frac{u\left(f_{C}\right)}{f_{C}}\right)^{2}+\left(\frac{u\left(f_{S}\right)}{f_{S}}\right)^{2}+\left(\frac{u\left(f_{E}\right)}{f_{E}}\right)^{2}+\left(\frac{u(K)}{K}\right)^{2}+\left(\frac{u(P)}{P}\right)^{2}} .
$$

\section{CONCLUSIONS}

This paper clearly shows that the principal features of MOSFET detectors can be successfully exploited to carry out absolute dosimetry in the context of a microSelectron HDR

${ }^{192}$ Ir source, even for situations where photon energy spectra are different from the reference location. The two major correction factors viz. change in the MOSFET response due to different photon spectra and dose accumulation, needed for assuring the quality of the dosimetric measurements, have been determined from appropriate measurements and Monte Carlo simulations. Apart from describing the methodology and the necessary correction factors, the present paper has included a detailed uncertainty analysis for the measured dose rates, thus providing sufficient confidence in the reported results. The experimental values obtained show a good agreement with the numerical simulations and almost 
all lie within the assessed uncertainties. The discrepancy between measured and calculated values for $82 \%$ and $95 \%$ of the experimental points lies below $5 \%$ and $10 \%$, respectively. It is advisable to use both types of sensors to cover a wide range of dose values and good to intercalibrate them to have a consistent check on the doses measured.

It may be mentioned that, although it is clearly preferable to physically employ the same detector as that for which the necessary correction factors have been measured and/or calculated, it should however be possible, with proper care and knowledge of the relative response, to employ other sufficiently similar MOSFETs. Furthermore, the improved design of MOSFETs to cover a wider range of shift in $V_{\text {th }}$ (i.e., higher accumulated dose values), as well as to provide a greater degree of energy independent response with innovative techniques such as compensation filters (method currently being developed at CMRP, Wollongong), could considerably enhance further the accuracy achievable with these dosimeters.

\footnotetext{
${ }^{a)}$ Electronic mail: omparkash.joneja@epfl.ch

${ }^{1}$ C. W. Scarantino, D. M. Ruslander, C. J. Rini, G. G. Mann, H. T. Nagle, and R. D. Black, "An implantable radiation dosimeter for use in external beam radiation therapy," Med. Phys. 31, 2658-2671 (2004).

${ }^{2}$ C. F. Chuang, L. J. Verhey, and P. Xia, "Investigation of the use of MOSFET for clinical IMRT dosimetric verification," Med. Phys. 29, 1109-1115 (2002).

${ }^{3}$ N. Jornet, P. Carrasco, D. Jurado, A. Ruiz, T. Eudaldo, and M. Ribas, "Comparison study of MOSFET detectors and diodes for entrance in vivo dosimetry in 18 MV x-ray beams," Med. Phys. 31, 2534-2542 (2004).

${ }^{4}$ P. Scalchi and P. Francescon, "Calibration of a MOSFET detection system for $6 \mathrm{MV}$ in vivo dosimetry," Int. J. Radiat. Oncol., Biol., Phys. 40, 987-993 (1998)

${ }^{5}$ R. Ramaseshan, K. S. Kholi, T. J. Zang, T. Lam, B. Norlinger, A. Hallil, and M. Islam, "Performance characteristics of a microMOSFET as an in vivo dosimeter in radiation therapy," Phys. Med. Biol. 49, 4031-4048 (2004).

${ }^{6}$ C. G. Rowbottom and D. A. Jaffray, "Characteristics and performance of a micro-MOSFET: An 'imageable' dosimeter for image-guided radiotherapy," Med. Phys. 31, 609-615 (2004).

${ }^{7}$ E. Brauer-Krisch, A. Bravin, M. Lerch, A. Rosenfeld, J. Stepanek, M. Di Michiel, and J. A. Laissue, "MOSFET dosimetry for microbeam radiation therapy at the European Synchrotron Radiation Facility," Med. Phys. 30,
}

583-589 (2003).

${ }^{8}$ D. J. Peet and M. D. Pryor, "Evaluation of a MOSFET radiation sensor for the measurement of entrance surface dose in diagonistic radiology," Br. J. Radiol. 75, 562-568 (1999).

${ }^{9}$ J. F. Briesmeister (Editor), "MCNP a general Monte Carlo N-particle transport code version 4C," Los Alamos National Laboratory Report, LA13709-M, 2000

${ }^{10}$ Organisation internationale de normalisation, Guide pour l'expression de l'incertitude de mesure, (ISBN 92-67-20188-3, 1995).

${ }^{11}$ A. B. Rosenfeld, M. L. F. Lerch, T. Kron, E. Brauer-Krisch, A. Bravin, A. Holmes-Siedle, and B. J. Allen, "Feasibility study of online high-spatialresolution MOSFET dosimetry in static and pulsed X-Ray radiation fields," IEEE Trans. Nucl. Sci. 48, 2061-2068 (2001).

${ }^{12}$ A. K. Quanch, J. Morales, A. B. Rosenfeld, and P. Metcalfe, "Measurement of radiotherapy X-ray skin dose on chest wall phantom," Med. Phys. 27(7), 1676-1680 (2000).

${ }^{13}$ J. F. Williamson and Z. Li, "Monte Carlo aided dosimetry of the microselectron pulsed and high dose-rate ${ }^{192}$ Ir sources," Med. Phys. 22(6), 809819 (1995).

${ }^{14}$ V. O. Zilio, O. P. Joneja, Y. Popowski, and R. Chawla, "Dosimetric characterization of radioactive seeds employed in prostate cancer therapy," Brachytherapy 3, 201-214 (2004).

${ }^{15}$ M. Soubra, J. Cygler, and G. Mackay, "Evaluation of a dual bias dual metal oxide-silicon semiconductor field effect transistor detector as radiation dosimeter," Med. Phys. 21, 567-572 (1994).

${ }^{16}$ T. Cheung, M. J. Butson, and P. K. N. Yu, "Effects of temperature variation on MOSFET dosimetry," Phys. Med. Biol. 49, N191-N196 (2004).

${ }^{17}$ V. O. Zilio, "Developpement et validation de method dosimetriques en ligne pour le traitment du cancer de la prostate," These No. 3267 2005, Ecole Polytechnique Federale de Lausanne.

${ }^{18}$ V. O. Zilio, O. P. Joneja, Y. Popowski, F. O. Bochud, and R. Chawla, ${ }^{\prime \prime}{ }^{144} \mathrm{Ce}$ as a potential candidate for interstitial and intravascular brachytherapy,” Int. J. Radiat. Oncol., Biol., Phys. 62(2), 585-594 (2005).

${ }^{19}$ C. R. Edwards, S. Green, J. E. Palethorpe, and P. J. Mountford, "The response of a MOSFET, $p$-type semiconductor and LiF TLD to quasimonoenergetic x-rays," Phys. Med. Biol. 42, 2383-2391 (1997).

${ }^{20}$ T. Kron, L. Duggan, T. Smith, A. Rosenfeld, M. Butson, G. Kaplan, S. Howlett, and K. Hyodo, "Dose response of various radiation detectors to synchrotron radiation," Phys. Med. Biol. 43, 3235-3259 (1998).

${ }^{21}$ B. Wang, C.-H. Kim, and X. G. Xu, "Monte Carlo modelling of a HighSensitivity MOSFET dosimeter for low- and medium-energy photon sources," Med. Phys. 31, 1003-1008 (2004).

${ }^{22}$ C. M. Dozier and D. B. Brown, "Effect of photon energy on the response of MOSFET devices," IEEE Trans. Nucl. Sci. 28(6), 4137-4141 (1981).

${ }^{23}$ D. M. Long, D. G. Millward, and J. Walace, "Dose enhancement effect in semiconductor devices," IEEE Trans. Nucl. Sci. 29(6), 1980-1984 (1982). 